\title{
METABOLIC CHANGES DURING DEVELOPMENT OF WALKER-256 CARCINOSARCOMA RESISTANCE TO DOXORUBICIN
}

\author{
I.N. Todor*, N.Yu. Lukianova*, Yu.V. Shvets, Yu.V. Lozovska, V.F. Chekhun \\ R.E. Kavetsky Institute of Experimental Pathology, Oncology and Radiobiology, NAS of Ukraine, Kyiv \\ 03022, Ukraine
}

\begin{abstract}
Aim: To study indices of energy metabolism, content of $\mathrm{K}^{+}$and $\mathrm{Mg}^{++}$both in peripheral blood and in Walker-256 carcinosarcoma during development of resistance to doxorubicin. Methods: Resistance of Walker-256 carcinosarcoma to doxorubicin has been developed through 12 subsequent transplantations of tumor after the chemotherapy. Parental strain was inhibited by drug by $65 \%$, while transitional resistant substrains - by $\mathbf{3 0} \%$ and $2 \%$, respectively. Determination of biochemical indices in blood serum and homogenates of tumor tissue, level of potassium, magnesium, lactate, glucose, activities of lactate dehydrogenase and glucose6-phosphate dehydrogenase was performed with the help of biochemical and immune-enzyme analyzer GBG ChemWell 2990 (USA) using standard kits. Polarography was used to determine indices of mitochondrial oxidative phosphorylation. Study of mitochondrial membrane potential was carried out on flow cytometer Beckman Coulter Epics XL using dye JC-1. Results: It has been determined that development of drug resistance causes the decrease of $\mathrm{K}^{+}, \mathrm{Mg}^{++}$, glucose content in blood serum and increase of these indices in tumor tissue. At the same time, gradual tumor's loss of sensitivity is characterized by decrease of glycolysis activity in it and activation of mitochondrial oxidative phosphorylation and pentose phosphate pathway of glucose degradation, which causes more intensive formation of NADPH. Conclusion: Development of drug resistance of tumor causes certain metabolic changes in organism and tumor. Further study of such changes will make possible to determine tumor and extratumor markers of resistance. Key Words: tumor, drug resistance, potassium, magnesium, glycolysis, pentose phosphate cycle, mitochondrial membrane potential.
\end{abstract}

Study of pathogenesis of malignant neoplasms both in experimental and clinical observations has a great theoretical and practical significance. While searching metabolic differences between normal and tumor cells, researchers have paid attention first of all to those processes, which underlie intensity of growth and division of cells, and, primarily processes of biosynthesis of nucleic acids and anaerobic and aerobic degradation of carbohydrates - glycolysis and mitochondrial oxidative phosphorylation. At that time, significant changes in important metabolic systems have been observed in tumor cells [1-7]. The cause of such changes is the loss by tumor cell of some regulatory mechanisms, which act on genetic or metabolic level.

Significant role in metabolism of cells is played by water-salt metabolism [8-10]. Properties of cells and their viability also depend on appropriate ion concentrations. Consumption of glucose by cells is always accompanied with transfer of potassium from intercellular liquid inside cell [9]. In general, biochemical reactions require certain composition of medium and maintenance of appropriate water-salt balance both in tumor and in organism. This issue becomes especially important, when it is spoken of occurrence and development of drug resistance of malignant tumors. For this reason, in this work we have studied important bioenergetics indices, content of $\mathrm{K}^{+}$and

Submitted: December 21, 2014.

*Correspondence: E-mail: todor_igor@yahoo.com;

Lu_na_u@rambler.ru;

Fax: 38(044)258-16-56

Abbreviations used: ADP - adenosine 5'-diphosphate; NADP - $\beta$-nicotinamide adenine dinucleotide phosphate; $\mathrm{NADPH}-\beta$-nicotinamide adenine dinucleotide phosphate reduced; $\Delta \psi_{\mathrm{m}}$ - the mitochondrial transmembrane electrochemical gradient.
$\mathrm{Mg}^{++}$in both peripheral blood and Walker-256 carcinosarcoma during development of its resistance to doxorubicin.

\section{MATERIALS AND METHODS}

Experimental model. In the study, female rats weighting $120-150 \mathrm{~g}$ bred in the vivarium of the R.E. Kavetsky Institute of Experimental Pathology, Oncology and Radiobiology, NAS of Ukraine (Kyiv, Ukraine), were used. As experimental model, Walker-256 carcinosarcoma was chosen. Tumor transplantation was performed by subcutaneous injection of $20 \%$ Walker-256 carcinosarcoma cell suspension in the thigh area. Animals were housed as the control (N1) and experimental (N2) groups (nor less than 10 animals per group). When the volume of tumor reached $0.5 \mathrm{~cm}^{3}$, six doxorubicin (Ebewe, Austria) injections $(1.5 \mathrm{mg} / \mathrm{kg}$ daily) were administered to the animals of the group 2. Then the animals were decapitated under narcosis (Sedazin, Biowet-Pulawy, Poland) according to the rules of Ethic Committee; tumors were resected, 20\% tumor cell suspension was prepared and transplanted to the animals from the new control and experimental groups. Then doxorubicin therapy was performed by the same schedule. In total, 12 courses of tumor transplantation/doxorubicin therapy were performed.

All studies have been performed during development of resistance of Walker-256 carcinosarcoma to doxorubicin. Animals, which underwent removal of tumor for the study, have not received cytostatic drug directly. Indices of ionic homeostasis and bioenergetics of parental Walker-256 carcinosarcoma (inhibition of growth by doxorubicin has constituted $65.0 \pm 5.8 \%$ ) and resistant variants of this tumor (inhibition of growth has constituted $30.0 \pm 2.7 \%$ and $2.1 \pm 0.5 \%$, respectively) have been studied. 
Mitochondria study. Oxidative phosphorylation indices of tumor cell mitochondria were determined according [11]. The mitochondrial transmembrane electrochemical gradient $\left(\Delta \psi_{\mathrm{m}}\right)$ was measured using JC1-staining [12].

Analysis of mitochondrial transmembrane potential. The mitochondrial transmembrane electrochemical gradient $\left(\Delta \psi_{\mathrm{m}}\right)$ was measured using JC-1. JC-1, a cell permeable, cationic, lipophilic dye freely crosses the mitochondrial membrane and forms $\mathrm{J}$-aggregates which fluoresce red; accordingly, viable cells with a normal mitochondrial membrane potential when stained with JC-1 exhibit a pronounced orange fluorescence (FL2). Following an apoptotic stimulus, the resultant decrease in the mitochondrial membrane potential prevents $\mathrm{JC}-1$ from entering the mitochondria and remains as monomers in the cytosol that emits a predominantly green fluorescence (FL1). Therefore, the ratio of J-aggregates/monomers serves as an effective indicator of the cellular mitochondrial transmembrane potential, allowing apoptotic cells to be easily distinguished from their non-apoptotic counterparts. Briefly, Walker-256 cells $\left(2.5 \cdot 10^{5} / \mathrm{ml}\right)$ were stained with $\mathrm{JC}-1$ ( $7.5 \mathrm{mM}$ in PBS, $10 \mathrm{~min}, 37^{\circ} \mathrm{C}$ ). Cells were then acquired in a flow cytometer on the basis of quadrant plot to distinguish monomers from $\mathrm{J}$-aggregates. To set the quadrants, cells were treated with $\mathrm{H}_{2} \mathrm{O}_{2}\left(20 \mathrm{mM}, 37^{\circ} \mathrm{C}, 30 \mathrm{~min}\right)$, representative of cells with depolarized mitochondrial membrane potential. The flow cytometer "Coulter Epics XL" (Beckman Coulter, USA) was used in studies.

Determination of biochemical indices in blood serum and homogenates of tumor tissue of the Walker-256 carcinosarcoma, level of potassium, magnesium, lactate, glucose, activities of lactate dehydrogenase and glucose-6-phosphate dehydrogenase has been performed with the help of biochemical and immune-enzyme analyzer "GBG ChemWell 2990" (GBG, USA) using standard kits for this equipment.

Statistical processing of the data was performed by the use of the software Statistica (v. 7.0) and Student's $\boldsymbol{t}$-criterion. A statistically significant difference was considered to be present at $p<0.05$.

\section{RESULTS AND DISCUSSION}

As stated above, the problem of drug resistance of malignant tumors is one of the most significant in oncology. For this reason, in our studies we primarily have paid attention to the processes of glycolysis and oxidative phosphorylation, which are the main sources of energy and plastic material for biosynthetic reactions of cell [13]. On the other hand, during the development of drug resistance, we have determined level of some ions, which characterize watersalt metabolism of cells, both in organism and tumor. The main biological role of electrolytes consists in their osmotic action, in maintenance of electrical neutrality in biological fluids and tissues of organism and in their impact on enzyme reactions in cells. At the same time, first of all we have evaluated level of $\mathrm{K}^{+}$and $\mathrm{Mg}^{++}$in blood serum and tumor in the process of induction of doxorubicin resistance of the Walker-256 carcinosarcoma. $\mathrm{K}^{+}$ is one of the key element of water-salt metabolism. Its level in organism significantly increases when malignant process occurs. Moreover, tumor tissue is also characterized by increased concentration of potassium ions [9].

Magnesium is one of the key cations required for enzyme reactions $[8,13]$. For instance, for the activity of DNA-polymerase and phosphofructokinase, allosteric enzyme of glycolysis, ions of $\mathrm{Mg}^{++}$are highly required. In general, magnesium is required for reactions with assistance of most of phosphokinases.

In the result of carried out studies, the following data concerning concentration of potassium ions in peripheral blood serum have been obtained (Fig. 1). It has been determined that in blood serum of rats with parental Walker-256 carcinosarcoma, concentration of $\mathrm{K}^{+}$was significantly higher, than in intact (control) animals that corresponds with data of literature [9]. However, during development of resistance of tumor to doxorubicin, concentration of potassium ions in serum starts gradually decreasing, and in tumor, though insignificantly, but gradually increasing (see Fig. 1). This is probably connected with the fact that resistant tumor requires more $\mathrm{K}^{+}$and more impacts the water-salt metabolism in organism, than parental (more sensitive to doxorubicin) Walker-256 carcinosarcoma. Probably, content of water in organism with resistant tumor is higher, than in organism with sensitive tumor.

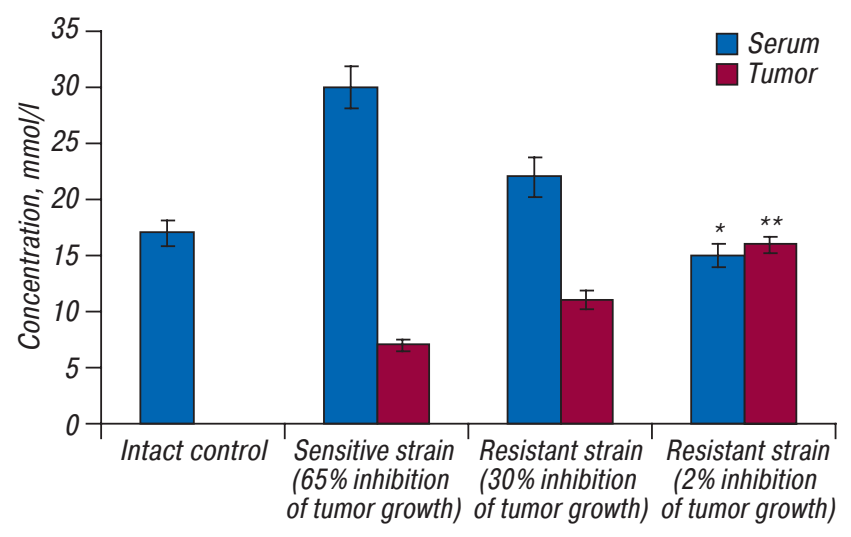

Fig. 1. Potassium levels in blood serum and tumor tissue supernatant of animals with Walker-256 carcinosarcoma during development of resistance to doxorubicin ( ${ }^{*} p<0.05$ compared with sensitive tumor-bearing rats; ${ }^{* *} p<0.05$ compared with sensitive tumor)

The same data have been obtained concerning concentration of magnesium ions. Concentration of $\mathrm{Mg}^{++}$ in blood serum of animals with sensitive Walker-256 carcinosarcoma is also higher, than in intact rats (Fig. 2). This indicates that malignant process significantly changes electrolyte balance in organism. However, with development of tumor resistance to doxorubicin, content of this cation in blood serum was gradually decreasing, and in tumor - gradually increasing. As stated above, $\mathrm{Mg}^{++}$ is required for many enzyme reactions associated with phosphorylation and DNA synthesis. About activation of intracellular metabolism in the process of development of malignant tumors drug resistance we pointed out in our previous studies [14].

Development of resistance of Walker-256 carcinosarcoma to doxorubicin is also characterized by changes in energy metabolism. Firstly, we have studied, how level 
of glucose is changed in blood serum of animals. It has been determined that level of this compound in blood serum of tumor host is significantly lower, than in intact rats (Fig. 3). Mentioned fact is the consequence of the fact that malignant tumor is characterized trap of glucose. As the development of resistance progressed, level of glucose in blood serum of animals even greater decreased. However, this index in tumor, while gradually losing sensitivity to the cytostatic drug, on the contrary, was increasing (see Fig. 3). This logically corresponds with dynamics of change of activity of lactate dehydrogenase both in blood serum and, especially, Walker-256 carcinosarcoma (Fig. 4). On the background of reduction of lactate dehydrogenase activity and, correspondingly, level of lactate (Fig. 5), the increase of glucose in tumor was observed. Such changes in mentioned indices indicate gradual decrease of glycolysis activity in tumor cells during development of resistance to doxorubicin. At that pyruvate is most likely metabolized in Krebs cycle and to a lesser degree is used for the formation of lactate. Such metabolic situation can cause the activation of generation of Acetyl-CoA that, in turn, stimulates increase of cholesterol that corresponds with the results of our previous studies $[15,16]$.

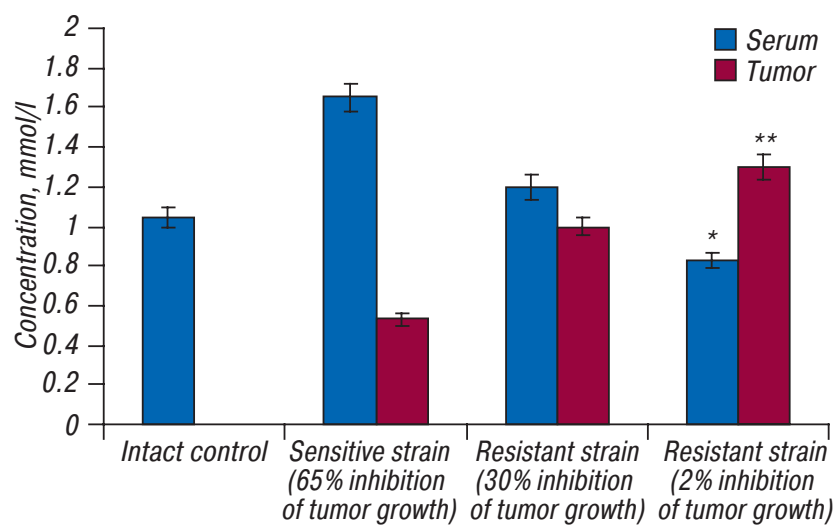

Fig. 2. Magnesium levels in blood serum and tumor tissue supernatant of animals with Walker-256 carcinosarcoma during development of resistance to doxorubicin ( ${ }^{*} \mathrm{p}<0.05$ compared with sensitive tumor-bearing rats; ${ }^{* *} p<0.05$ compared with sensitive tumor)

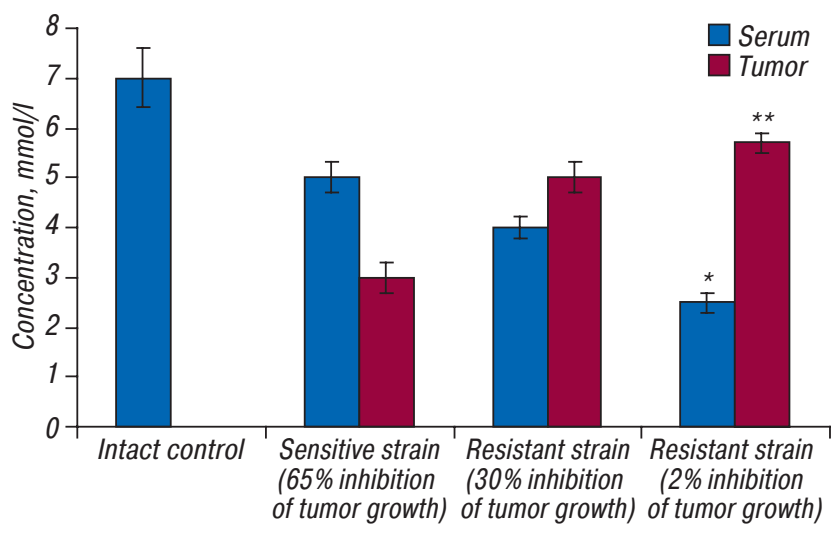

Fig. 3. Glucose levels in blood serum and tumor tissue supernatant of animals with Walker-256 carcinosarcoma during development of resistance to doxorubicin ( ${ }^{*} p<0.05$ compared with sensitive tumor-bearing rats; ${ }^{* *} p<0.05$ compared with sensitive tumor)

Since we studied intensity of glycolysis in tumor cells, it would be logical to study also functional activity of mitochondria. To solve this question we have used two methodic approaches: polarography (oxygen consumption by mitochondria) and application of dye JC-1. Data of the studies are represented in Fig. 6 and 7. As seen in Fig. 6, development of resistance ofWalker-256 carcinosarcoma to doxorubicin causes the decrease of content of monomer form JC-1 in tumor cells (FL1; green fluorescence). It indicates higher membrane potential of mitochondria and increase of functional activity of these organelles. These results correspond well with data of polarographic determination of oxygen consumption rate by mitochondria. As seen in Fig. 7, development of resistance to doxorubicin causes increase of oxygen consumption rate by organelles at phosphorylation of exogenous ADP in the presence of glutamate. However, it should be mentioned that despite changes of bioenergetics indices in development of drug resistance, intensity of glycolysis in tumor cells remains high, and intensity of mitochondrial oxidative phosphorylation - low compared with nontransformed cells.

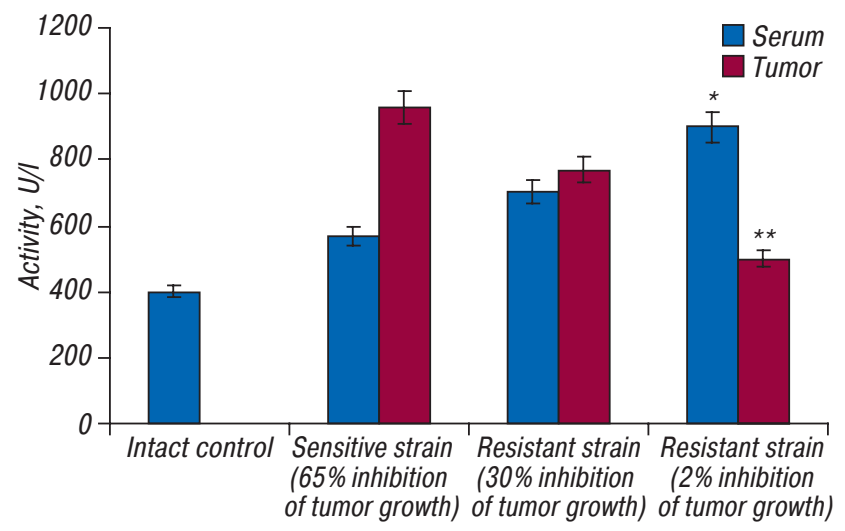

Fig. 4. Lactate dehydrogenase activity in blood serum and tumor tissue supernatant of animals with Walker-256 carcinosarcoma during development of resistance to doxorubicin ( ${ }^{*} p<0.05 \mathrm{com}$ pared with sensitive tumor-bearing rats; ${ }^{* \star} p<0.05$ compared with sensitive tumor)

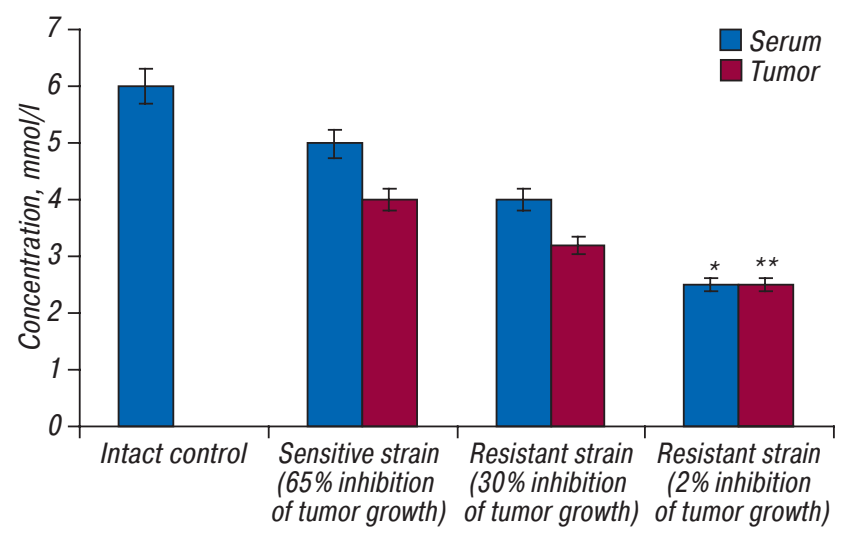

Fig. 5. Lactate levels in blood serum and tumor tissue supernatant of animals with Walker-256 carcinosarcoma during development of resistance to doxorubicin $\left({ }^{*} \mathrm{p}<0.05\right.$ compared with sensitive tumor-bearing rats; ${ }^{*} p<0.05$ compared with sensitive tumor)

Since glucose is known to be able also to degrade by pentose phosphate pathway [13], we have studied activity of the first enzyme of this cycle - glucose6 -phosphate dehydrogenase. It has been determined that as development of drug resistance in Walker-256 carcinosarcoma progresses, gradual increase of activity of this enzyme occurs (Fig. 8). It is very important that NADP is co-enzyme for this enzyme. Pentose phosphate cycle plays double role in the formation of NADPH. In two stages of pentose phosphate cycle, oxidation of one glu- 
cose molecule occurs and twelve NADPH molecules are formed. This component does not participate in cellular oxidation, but is required for the specific reduction reactions, among which are reduction of glutathione, biosynthesis of fatty acids and different steroid hormones [1719]. Pentose phosphate pathway is especially important for those organs and cells, in which these reactions are actively proceeding - adipocytes, liver, mammary gland, adrenal gland cortex. Moreover, regulation of water-salt metabolism is tightly connected with activity of pentose phosphate cycle. On the other hand, formation of important metabolites takes place in cycle and especially ribose-5-phosphate, which is precursor of nucleic acids.

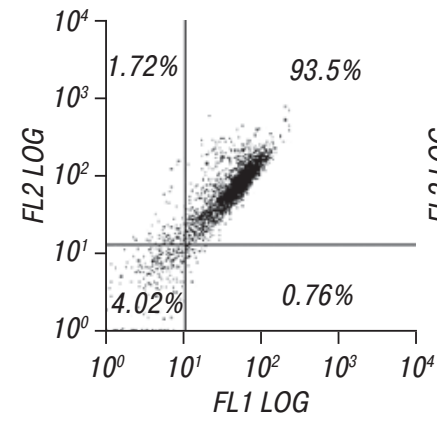

Sensitive strain (65\% inhibition of tumor growth)

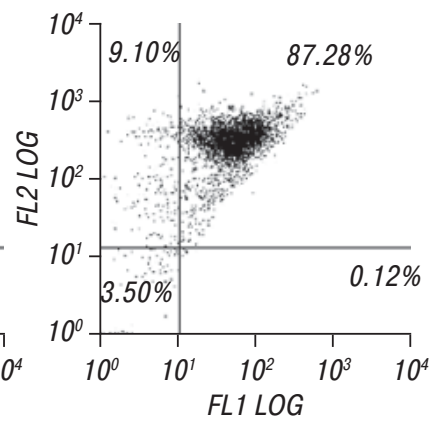

Resistant strain (2\% inhibition of tumor growth)

Fig. 6. The mitochondrial transmembrane electrochemical gradient $\left(\Delta \psi_{\mathrm{m}}\right)$ (JC-1 staining) in Walker-256 carcinosarcoma during development of resistance to doxorubicin

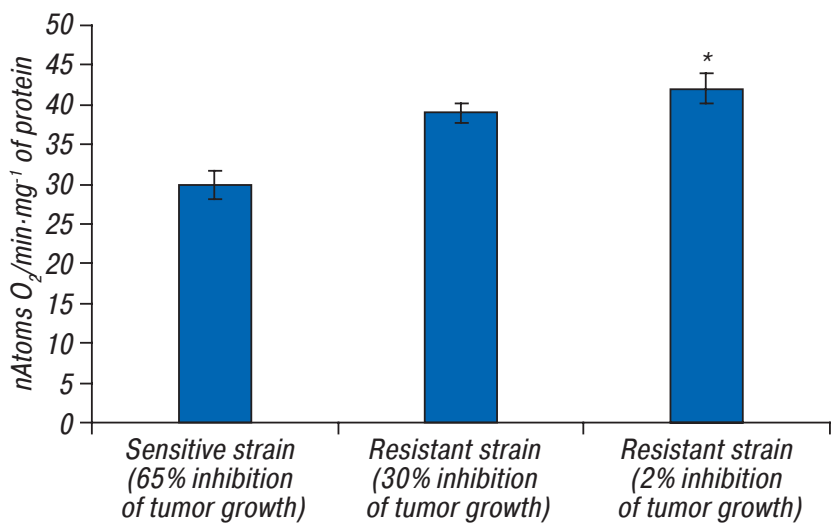

Fig. 7. Oxygen consumption by tumor cell mitochondria during exogenous ADP phosphorylation (oxidation substrate - glutamate) ( ${ }^{\star} p<0.05$ compared with sensitive tumor)

Thus, obtained data show significant changes in content of $\mathrm{K}^{+}$and $\mathrm{Mg}^{++}$and indices of energy metabolism in blood serum and in tumor cells during resistance development of Walker-256 carcinosarcoma to doxorubicin. Results of studies show that as development of drug resistance in blood serum of tumor host progresses, decrease of $\mathrm{K}^{+}, \mathrm{Mg}^{++}$, glucose and increase of these indices in tumor tissue are observed. At that gradual tumor's loss of sensitivity is characterized by decrease of glycolysis activity in it and activation of mitochondrial oxidative phosphorylation and pentose phosphate way of glucose degradation, which causes more intensive formation of NADPH. Reduced NADP is required for many reduction reactions that is very important for protection of tumor cells from damaging agents.

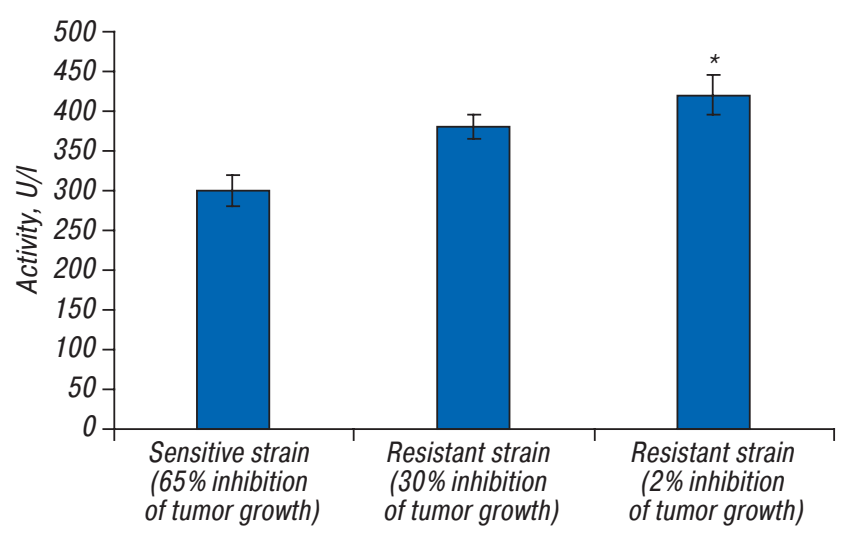

Fig. 8. Glucose-6-phosphate dehydrogenase activity inWalker-256 carcinosarcoma supernatant during development of resistance to doxorubicin ( ${ }^{\star} \mathrm{p}<0.05$ compared with sensitive tumor)

\section{REFERENCES}

1. Pedersen PL. Tumor mitochondria and the bioenergetics of cancer cells. Prog Exp Tumor Res 1978; 22: 190-274.

2. Matsuno T. Bioenergetics of tumor cells: glutamine metabolism in tumor cell mitochondria. Int J Biochem 1987; 19: 303-7.

3. Debarardinis RJ, Cheng T. The diverse function of glutamine in metabolism, cell biology and cancer. Oncogene 2010; 29: 313-24.

4. Lange SS, Takata K, Wood RD. DNA polymerases and cancer. Nat Rev Cancer 2011; 11: 96-110.

5. Lin J, Lee I-M, Song Y. Plasma homocysteine and cysteine and risk of breast cancer in women. Cancer Res 2013; 70: 2397-405.

6. Nowotarski SL, Woster PM, Casero Jr RA. Polyamines and cancer: implications for chemoprevention and chemotherapy. Expert Rev Mol Med 2013; 15: e3 (21 p.).

7. Torti SV, Torti FM. Cellular iron metabolism in prognosis and therapy of breast cancer. Crit Rev Oncog 2013; 18: 435-48.

8. Vasiliev YuM. Relationships of tumor cells with each other and normal cells. In: The biology of malignant growth. Moscow, 1965: 200-19 (in Russian).

9. Pashinsky VG. The water - salt metabolism and cancer. Tomsk: Tomsk University Press, 1981. 265 p. (in Russian).

10. Kellum J, Cerda J. Renal and metabolic disorders. Pittsburgh Critical Care Medicine: Oxford University Press, 2013

11. Todor IN, Chekhun VF. The respiration of mitochondria from the liver of rats treated with cisplatinum and millimeter range electromagnetic radiation. Exp Oncol 1995; 17: 137-40.

12. Manna A, Saha P, Sarker A, et al. Malabaricone-A induces a redox imbalance that mediates apoptosis in U937 cell line. PLoS ONE 2012; 7: e36938.

13. Nelson DL, Cox MM. Lehninger principles of biochemistry (4th ed.). 2004.

14. Yurchenko OV, Todor IN, Tryndyak VP, et al. Resistance of Guerin's carcinoma cells to cisplatin: biochemical and morphological aspects. Exp Oncol 2003; 25: 64-8.

15. Todor IN, Lukyanova NYu, Chekhun VF. The lipid content of cisplatin- and doxorubicin-resistant MCF-7 human breast cancer cells. Exp Oncol 2012; 34: 97-100.

16. Chekhun VF, Naleskina LA, Todor IN, et al. Alteration in lipid composition of plasma membranes of sensitive and resistant Guerin carcinoma cells due to the action of free and liposomal form of cisplatin. Exp Oncol 2013; 35: 192-7.

17. Estrela M, Ortega A, Obrador E. Glutathione in cancer biology and therapy. Crit Rev Clin Lab Sci 2006; 43: 143-81.

18. Montero AJ, Jassem J. Cellular redox pathways as a therapeutic target in the treatment of cancer. Drugs 2011; 71: 1385-96.

19. Traverso N, Ricciarelli R, Nitti M, et al. Role of glutathione in cancer progression and chemoresistance. Oxid Med Cell Longev 2013; 2013; dx.doi.org/10.1155/2013/972913, ID 972913 (10 p.). 